\title{
Ground-based CCD astrometry with wide-field imagers
}

\section{A star catalog for M 67: WFI@2.2 m MPG/ESO astrometry, FLAMES@VLT radial velocities $\star, \star \star, \star \star \star$}

\author{
R. K. S. Yadav ${ }^{1, \dagger}$, L. R. Bedin ${ }^{2}$, G. Piotto ${ }^{1}$, J. Anderson ${ }^{2}$, S. Cassisi ${ }^{3}$, S. Villanova ${ }^{4}$, \\ I. Platais ${ }^{5}$, L. Pasquini ${ }^{6}$, Y. Momany ${ }^{7}$, and R. Sagar $^{8}$
}

1 Dipartimento di Astronomia, Universita di Padova, Vicolo dell' Osservatorio 2, 35122 Padova, Italy e-mail: [giampaolo.piotto; sandro.villanova]@unipd.it

2 Space Telescope Science Institute, 3700 San Martin Drive, Baltimore, MD 21218, USA e-mail: [bedin; jander]@stsci .edu

3 INAF - Osservatorio Astronomico di Collurania, via M. Maggini, 64100 Teramo, Italy e-mail: cassisi@oa-teramo.inaf.it

${ }^{4}$ Grupo de Astronomía, Departamento de Física, Universidad de Concepción, Casilla 160-C, Concepción, Chile e-mail: svillanova@astro-udec.cl

5 Dept. of Physics and Astronomy, The Johns Hopkins University, Baltimore, MD 21218, USA e-mail: imants@pha.jhu.edu

${ }^{6}$ European Southern Observatory, Garching, Karl-Schwarzschild-Str. 2, 85748 Germany e-mail: lpasquin@eso.org

7 INAF - Osservatorio Astronomico di Padova, Vicolo dell'Osservatorio 5, 35132, Italy e-mail: yazan.almomany@oapd.inaf.it

8 Aryabhatta Research Institute of Observational Sciences, Manora Peak, Nainital 263 129, India

e-mail: sagar@aries.ernet.in

Received 14 December 2007 / Accepted 25 February 2008

\begin{abstract}
Context. The solar-age open cluster M 67 (C0847+120, NGC 2682) is a touchstone in studies of the old Galactic disk. Despite its outstanding role, the census of cluster membership for M 67 at fainter magnitudes and their properties are not well-established.

Aims. Using proprietary and archival ESO data, we have obtained astrometric, photometric, and radial velocities of stars in a $34 \times 33 \operatorname{arcmin}^{2}$ field centered on the old open cluster M 67 .

Methods. The two-epoch archival observations separated by 4 years and acquired with the Wide-Field Imager at the $2.2 \mathrm{~m} \mathrm{MPG/ESO}$ telescope were reduced with our new astrometric techniques, as described in the first paper in this series. The same observations served to derive calibrated BVI photometry in M 67. Radial velocities were measured using the archival and new spectroscopic data obtained at the VLT.

Results. We have determined relative proper motions and membership probabilities for $\sim 2400$ stars. The precision of proper motions

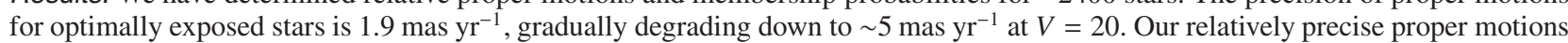
at $V>16$ were first obtained in this magnitude range for M 67. Radial velocities were measured for 211 stars in the same field. We also present a detailed comparison with recent theoretical isochrones from several independent groups.

Conclusions. For the M 67 area, we provide positions, calibrated BVI photometry, relative proper motions, membership probabilities, and radial velocities. We demonstrate that ground-based CCD mosaic observations just a few years apart are producing proper motions, allowing reliable membership determination. We have produced a catalog that is electronically available to the astronomical community.
\end{abstract}

Key words. Galaxy: open clusters and associations: individual: M 67 - astrometry - catalogs

\section{Introduction}

^ Based on observations with the MPG/ESO $2.2 \mathrm{~m}$ and ESO/VLT telescopes, located at La Silla and Paranal Observatory, Chile, under DDT program 278.D-5027, and the archive material.

$\star \star$ This is paper 33 of the WIYN Open Cluster Study (WOCS).

$\star \star \star$ Full Table 5 is only available in electronic form at the CDS via anonymous ftp to cdsarc.u-strasbg.fr (130.79.128.5) or via

http://cdsweb.u-strasbg.fr/cgi-bin/qcat?]/A+A/484/609

$\dagger$ present affiliation: Aryabhatta Research Institute of Observational Sciences, Manora Peak, Nainital 263 129, India

e-mail: rkant@aries.ernet.in
Open clusters are the basis of our understanding of stellar evolution. Among them, M $67(\mathrm{C} 0847+120)$ plays a special role. Due to their dynamical evolution and inevitable dissolution, old open clusters are rare. In the handful of known open clusters with ages comparable to or older than the Sun, M 67 also has solar chemical composition, is relatively nearby $(\sim 900 \mathrm{pc})$, and has low interstellar reddening, so it is one of the most studied open clusters (see Table 1 for fundamental parameters). Among these studies we recall determinations of the cluster proper motion membership (Sanders 1977; Girard et al. 1989; Zhao et al. 1993), 
Table 1. Fundamental parameters of M 67.

\begin{tabular}{ccc}
\hline \hline Parameter & Value & Reference \\
\hline$\alpha(\mathrm{J} 2000)$ & $08^{\mathrm{h}} 51^{\mathrm{m}} 23^{\mathrm{s}} .3$ & this work \\
$\delta(\mathrm{J} 2000)$ & $+11^{\circ} 49^{\prime} 02^{\prime \prime}$ & this work \\
$l$ & $215^{\circ} .688$ & this work \\
$b$ & 31.923 & this work \\
{$[\mathrm{Fe} / \mathrm{H}]$} & $+0.03 \pm 0.01$ & Randich et al. $(2006)$ \\
$E(B-V)$ & 0.041 & Taylor $(2007)$ \\
$(m-M)$ & $9.56-9.72$ & this work \\
Age $[\mathrm{Gyr}]$ & $3.5-4.8$ & this work \\
\hline
\end{tabular}

photometry (Montgomery et al. 1993; Sandquist 2004; Momany et al. 2001; Balaguer-Núñez et al. 2007), and high-resolution spectroscopy to determine chemical composition (Tautvaišiene et al. 2000; Randich et al. 2006).

In addition, M 67 harbors an array of interesting objects: blue stragglers (Shetrone \& Sandquist 2000), one AM Her star (Gilliland et al. 1991; Pasquini et al. 1994), several X-ray active stars, detected as coronal sources by ROSAT (Pasquini \& Belloni 1998; van den Berg et al. 1999), a red straggler, and two subsubgiants (Mathieu et al. 2003). Other studies have been devoted to the white dwarf cooling sequence (Richer et al. 1998), the evolution of chromospheric activity along the red giant branch (Dupree et al. 1999) and in solar-type stars (Pace \& Pasquini 2004), and the evolution of angular momentum of stars close to the turnoff (Melo et al. 2001). The behavior of light elements such as lithium and beryllium and their implications for mixing theories have been studied by Pasquini et al. (1997); Jones et al. (1999) and Randich et al. (2007). Extensive radial velocity surveys have been carried out, resulting in the discovery of many binaries (e.g., Mathieu et al. 1997). Attempts have been made to determine stellar oscillations through a multi-site accurate photometry (Gilliland et al. 1991; Stello et al. 2007). Given the uniqueness of this cluster and the similarity of its key properties to the Sun, new studies are under way to explore the main sequence stars spectroscopically as has been done for the other clusters (Pace et al. 2007; Biazzo et al. 2007).

Despite the wealth of accumulated data on M 67, a fundamental question of complete cluster membership is still open. The existing high-quality proper motion surveys carried out by Sanders (1977); Girard et al. (1989) and Zhao et al. (1993) are based on photographic plates limited to $V \sim 16$. This is only three magnitudes below the main-sequence turnoff. However, dedicated and/or archival multi-epoch observations with wide-field CCD imagers offer new opportunities to derive precise proper motions within a time span of a few years, and they are also deeper by several magnitudes than the photographic surveys. The techniques of ground-based CCD astrometry (and photometry) have been developed and validated on two nearby globular clusters using the observations with the MPG/ESO 2.2 m telescope and its wide-fieldWFI@2.2 m camera (Anderson et al. 2006, hereafter Paper I). With this setup, just a 2.7 year time baseline yields proper motions as precise as $\sim 2 \mathrm{mas} \mathrm{yr}^{-1}$. The existing archival WFI@2.2 m CCD frames for M 67 made this open cluster a prime target for deep proper motions studies based on these techniques. There is one report on proper motions in M 67 for stars fainter than $V=16$ by Lattanzi (1993). No catalogs of data ever followed, we do not consider that this conference paper has contributed much to the membership studies of M 67. In this study we enlarge the number of stars with known proper motion determination by a factor of $\sim 2$ over
Table 2. Description of the WFI data sets used for this work.

\begin{tabular}{cccc}
\hline \hline Filters & Exposure time & Seeing & Airmass \\
\hline \multicolumn{4}{c}{ first epoch: Feb. 16, 2000 } \\
$B$ & $9 \times 30 \mathrm{~s}$ & $\sim 1^{\prime \prime} 0$ & 1.4 \\
$V$ & $9 \times 20 \mathrm{~s} ; 11 \times 30 \mathrm{~s} ; 3 \times 120 \mathrm{~s}$ & $\sim 1^{\prime \prime} 0$ & 1.5 \\
$I$ & $9 \times 30 \mathrm{~s}$ & $\sim 1^{\prime \prime} 0$ & 1.4 \\
\hline \multicolumn{4}{c}{ Second epoch: Feb. 12, 2004 } \\
$V$ & $1 \times 60 \mathrm{~s} ; 1 \times 300 \mathrm{~s}$ & $\sim 1^{\prime \prime} .2$ & 1.3 \\
\hline
\end{tabular}

a $0.3 \mathrm{deg}^{2}$ area down to $V \sim 21$, which is 5 mag deeper than the existing proper motion studies of M 67.

In addition, we analyzed a large number of FLAMES+GIRAFFE@VLT high-resolution spectra and determined radial velocities for 211 stars in the M 67 field that were accurate to $\sim 0.5 \mathrm{~km} \mathrm{~s}^{-1}$, the accuracy of which is suitable for reliable $R V$ membership and identification of short-period binary systems. All new observational data are provided to the astronomical community so that it is convenient for the follow-up studies.

The main goal of this study is to extend the membership analysis to much fainter magnitudes and present a catalog of objects in a wide-field $\left(\sim 30^{\prime} \times 30^{\prime}\right)$ centered on the old open cluster M 67. The catalog provides new positions, multi-band photometry, proper motions, membership probabilities, and radial velocities.

The structure of the paper is as follows. In Sect. 2 we describe observations and data reduction of both the images and spectra. In Sects. 3 and 4 we present the cluster proper motion membership analysis in two different ways. In Sect. 5 we compare them with other photometric data available in the literature. In Sect. 6 a detailed comparison with available models from different groups is given. Finally, in Sect. 7 we present and describe the electronic catalog and how to use it.

\section{Observational data and reductions}

\subsection{Astrometric and photometric reductions}

All the images used in this study were obtained with the WideField Imager (hereafter simply $W F I$ ) at the MPG/ESO $2.2 \mathrm{~m}$ telescope at La Silla, Chile. The WFI consists of eight $2048 \times$ 4096 EEV CCDs with a pixel scale of 238 milliarcsec (mas), which provides a total field-of-view of $34^{\prime} \times 33^{\prime}$. More details on the instrumental setup are given in Paper I.

To calculate proper motions, we searched the MPG/ESO $2.2 \mathrm{~m}$ telescope archive for suitable CCD imager frames providing deep images obtained at separate epochs. We found that the open cluster M 67 was observed in 2000 and 2004 during the technical nights. Some details of the WFI data set used in this work are given in Table 2. These observations contain short and long exposures, allowing both the red giant branch (RGB) and the relatively faint main-sequence (MS) stars to be sampled. It should be noted that these archival datasets were not taken with the goal of obtaining proper motions or well-calibrated photometry. The second-epoch is represented by only two images, and individual frames were not properly dithered. As a result, the distribution of registered stars is not contiguous. Additional CCD imaging is planned, however its success depends upon a proven record of publications.

We closely followed the prescription for reductions of WFI images given in Paper I. This includes standard manipulations with the pixel data such as de-biasing, flat-fielding, and correction for cosmic rays. At the core of fitting the star positions and 
fluxes is the empirical point spread function (PSF). In this concept, the PSF is represented by a look-up table on a very fine grid. It is well-documented that the shape of PSF changes with position on a chip. This variability can be captured by an array of PSFs across the chip. A fully automated code is developed to find appropriate stars to adequately represent the PSF. For all practical purposes the number of PSF stars per chip can vary from 1 to 15 , depending on the richness of a star-field. An iterative process has been designed to work from the brightest down to the faintest stars and find their precise position and instrumental flux. A reasonably bright star can be measured with a precision of $\sim 0.03$ pixel ( $\sim 7$ mas) from a single exposure. In this step of empirical PSF fitting, we obtained precise instrumental magnitudes in $B, V, I$ filters.

Another issue in astrometry with reflectors is the large geometric distortion in the focal plane that effectively changes the pixel scale across the field-of-view. There are different ways to derive this geometric distortion. We opted for a $9 \times 17$ element look-up table of corrections for each chip, derived from multiple optimally-dithered observations of the Galactic bulge in Baade's window (Paper I). This look-up table provides the best currently available characterization of geometrical distortions for the WFI@2.2 m. At any given location on the detector, a bi-linear interpolation between the four closest grid points from a look-up table provided the corrections for the target point. The derived look-up table may have a lower accuracy on the edges of a field because of the way the self-calibration frames were dithered (see Paper I). An additional source of uncertainty is related to a possible instability of distortions for the WFI@2.2 m reported earlier. This prompted us to use the localtransformation method to derive proper motions (see Paper I for details).

In the local transformation approach (a linear adjustment with six parameters, three in each of the two coordinates), a small set of local reference stars is selected around each target object. It is advantageous to use pre-selected cluster members to form a local reference frame because of a much lower intrinsic velocity dispersion among the cluster members (see Sect. 2.1.2). Then, a least-squares adjustment is used to translate the coordinates from one frame into another, taken at different epochs. The residuals of this transformation characterize relative proper motions convolved with measurement errors. In essence, this is a classical "plate pair" method but one extended to all possible combinations of the first and second-epoch frames. Relative proper motion of a target object is an average of all displacement measurements in its local reference frame. The last step is to estimate the measurement errors from intra-epoch observations where proper motions make a zero contribution. A complete description of all steps leading to proper motions is given in Paper I. We did not consider the effect of differential chromatic refraction because all CCD frames have been obtained within a narrow range of zenith distance (see Sect. 2.1.3 for details).

\subsubsection{Photometric calibration}

Initially, we transformed our instrumental magnitudes obtained in $B, V, I$ filters into the standard Johnson Kron-Cousins magnitudes using standard stars from Stetson's ${ }^{1}$ secondary standard star catalog (Stetson 2000, 2005). Unfortunately, the color and magnitude intervals covered by these secondary standards are not ideal for properly calibrating our sample. Our RGB stars are saturated, and the faint MS stars are not present in the

\footnotetext{
${ }^{1}$ http://cadcwww.hia.nrc.ca/standards/
}
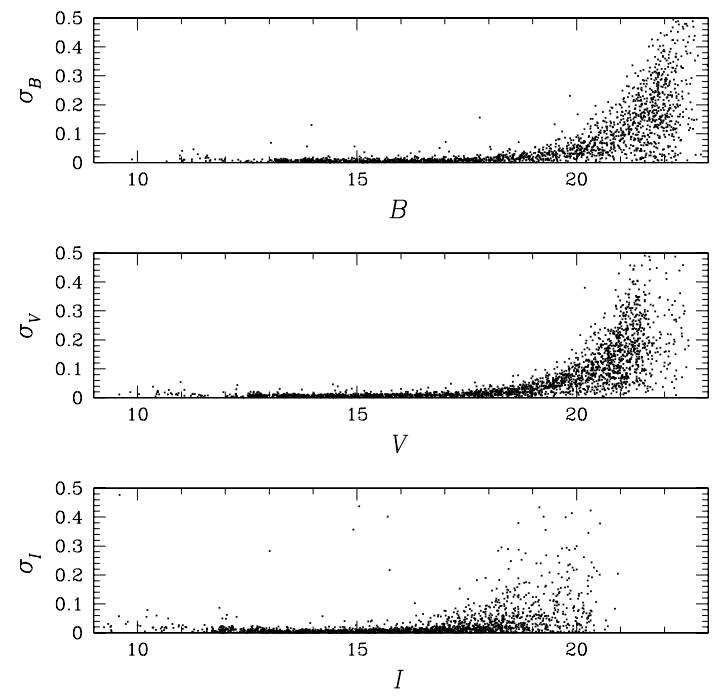

Fig. 1. Plot of error in magnitudes as a function of magnitude.

secondary standard catalog, thus confining us to a narrow range of colors for calibrations. For our calibrations, we therefore chose the high-precision $B V I$ photometry of M 67 by Sandquist (2004). This photometry, covering the entire color range of stars in M 67, is calibrated using the observations of Landolt's standards (Landolt 1992) and, to some extent, also Stetson's secondary standards. Sandquist (2004) also made use of photometric and spectroscopic data to select the objects most likely to be single stars. For these reasons and the ones outlined in Sect. 6, we link our photometry to that of Sandquist $(2004)^{2}$. This calibrated photometry covers only the inner fourWFI@2.2 m CCD chips. We used the common stars in dithered frames between the pairs of adjacent CCD chips to obtain individual photometric zero points in magnitude. Then, we solved for the global zero point and color term using one of the following transformation equations:

$$
\begin{aligned}
& B_{\mathrm{std}}=B_{\mathrm{ins}}+C_{b} *\left(B_{\mathrm{ins}}-V_{\mathrm{ins}}\right)+Z_{b} \\
& V_{\mathrm{std}}=V_{\mathrm{ins}}+C_{v} *\left(V_{\mathrm{ins}}-I_{\mathrm{ins}}\right)+Z_{v} \\
& I_{\mathrm{std}}=I_{\mathrm{ins}}+C_{i} *\left(V_{\mathrm{ins}}-I_{\mathrm{ins}}\right)+Z_{i}
\end{aligned}
$$

where $X_{\text {ins }}$ is the instrumental magnitude, $X_{\text {std }}$ Sandquist's calibrated magnitude, $C_{b}, C_{v}, C_{i}$ are the color terms (constants), and $Z_{b}, Z_{v}, Z_{i}$ are the global zero points. The quadratic color terms are negligible. Our color terms are consistent with those posted on the WFI@2.2 m webpage ${ }^{3}$. A comparison of our photometry with the Sandquist and Stetson catalog is presented in Sect. 5.

In Fig. 1 we show our photometric errors for each filter, as a function of the corresponding calibrated magnitude. The photometric errors (standard deviation) were computed from multiple observations, all reduced to the common photometric reference frame in the chosen bandpass. On average, the errors are more than $\sim 0.03 \mathrm{mag}$ for stars fainter than $V \sim 19 \mathrm{mag}$. The stars brighter than $V \sim 12.5$ mag show higher dispersion because of the image saturation.

\footnotetext{
${ }^{2}$ http://www.blackwellpublishing.com/products/ journals/suppmat/MNR7174/MNR7174sm.htm

${ }^{3}$ http://wWw. ls.eso.org/lasilla/sciops/2p2/E2p2M/WFI/ zeropoints/
} 


\subsubsection{Proper motions}

We first photometrically selected likely cluster members in the color-magnitude diagram and used only these stars as a local reference frame to transform the coordinates from one image into the system of the other image at a different epoch and, thus, derive relative proper motions. By predominantly using the cluster stars, we ensure that proper motions will be measured relative to the bulk motion of cluster stars. According to Girard et al.

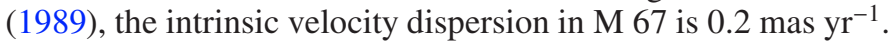
Over the four-year epoch difference that would result in a displacement of only 0.8 mas, which is smaller by a factor of 10 than the random measurement errors. Conversely, the tangential velocity dispersion of field stars is greater by a factor of $\sim 40$ than the cluster's intrinsic velocity dispersion. For field stars, proper motions clearly dominate over the measurement errors, and this has an adverse effect on the coordinate transformations. We iteratively removed some stars from the preliminary photometric member list that had proper motions clearly inconsistent with cluster membership, even though their colors placed them near the fiducial cluster sequence.

To minimize the influence of any unaccounted for residual distortion and refraction effects on proper motions, we used local transformations based on the nearest $\sim 20$ reference stars, typically extending over $\sim 300$ arcsec. These are well-measured cluster stars of any magnitude selected on the same CCD chip, as long as their preliminary proper motion is consistent with cluster membership. No systematics larger than our random errors are visible close to the corners or edges of chips. To avoid possible filter-dependent systematic errors, we measured proper motions only in the $V$ bandpass, for which the geometrical distortion corrections were originally derived (Paper I). Individual errors of proper motions for single stars were estimated as described in Sect. 7.3 of Paper I.

In a nutshell, we estimated the intra-epoch rms error from all first-epoch plates locally transformed into the same reference frame. Due to the lack of adequate statistics (too few images), we assumed that the second-epoch frames have the same errors for a given instrumental magnitude. The proper motion errors were computed as the rms of the proper motion, obtained from locally solving each first-epoch frame into second-epoch frame. These errors, however, are not totally independent because the same frame is used for a second epoch. Therefore, to obtain our best estimate of the proper motion standard error, we added in quadrature the initial proper motion error and the adopted error of the second-epoch image.

Figure 2 shows the distribution of standard errors $(\sigma)$ in proper motions for both coordinates as a function of $V$ magnitude. The precision of our proper motion measurement is $\lesssim 0.05$

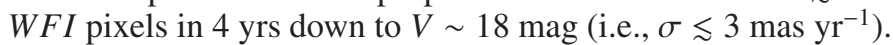
At fainter magnitudes, the errors gradually increase, reaching

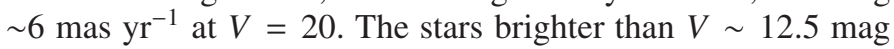
show a higher dispersion because of the image saturation.

\subsubsection{Astrometric calibration}

One of our goals is to provide an astrometric frame on the International Celestial Reference System (ICRS) in the area of M 67. Our astrometric reductions led us to the distortion-free cartesian coordinates of all objects. The positions of stars in each frame have been corrected for geometric distortion using a look-up table from Paper I, and then averaged into a common reference frame using a six-parameter linear transformation. The astrometric positions, of this catalog are still affected by

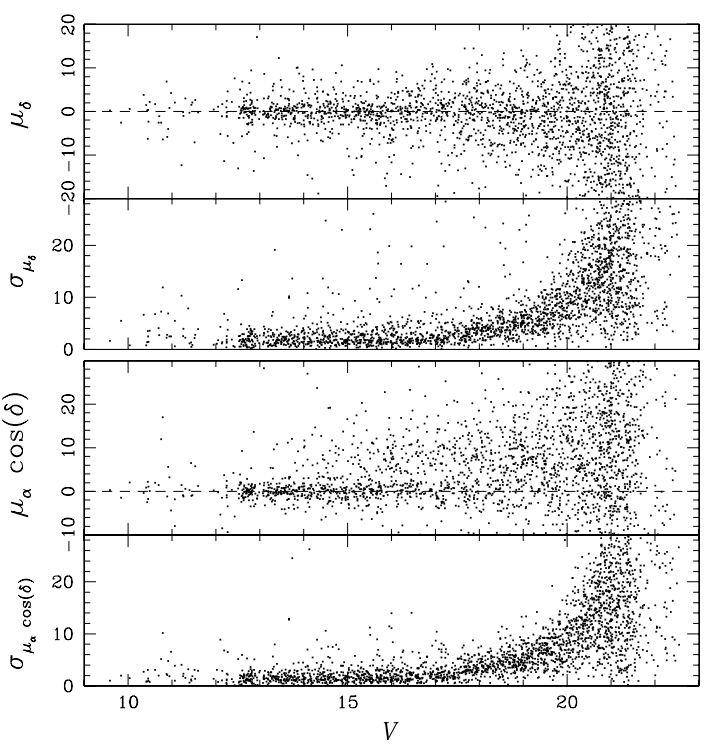

Fig. 2. Plots of proper motions and their standard deviations versus visual magnitude in mas $_{\mathrm{yr}^{-1}}$.

differential chromatic refraction (DCR) effects and the limitations on the stability of derived geometrical distortions, as described in Sect. 5.2 of Paper I.

To link the present M 67 catalog to ICRS, we used the UCAC2 catalog (Zacharias et al. 2004) as a reference frame. It was trimmed down to the stars with positional accuracies better than 75 mas. Owing to the excellent geometric distortion solution and a reasonable stability of the intra-chip positions it was possible to apply a single-plate model involving linear and quadratic terms and a small but significant cubic term in each coordinate. This solution also absorbs effects of differential refraction but not its chromatic component. The standard error of equatorial solution is only 30 mas in each coordinate, thus indicating the high potential of the WFI for astrometry. A total of 489 reference stars were used in this solution. The provided J2000 coordinates in Table 5 are for the epoch 2000.13.

A compilation of kinematic and photometric data from a variety of sources (Platais, unpublished) allowed us to pre-select a fairly clean list of cluster members down to $V \sim 16$, which then was used to derive a better geometrical center of M 67. The marginal distributions of cluster members along right ascension and declination were fit with a Gaussian. This fit yielded a cluster center listed in Table 1, accurate to about $3^{\prime \prime}$.

The proper motions in this work have not been corrected for differential chromatic refraction effects (DCRE) as in Paper I. Nonetheless, we verified the differential chromatic refraction effect and found it to be negligible in this data set owing to the very similar observing conditions at both epochs.

The only determination of absolute proper motion for M 67 is the one from using two Hipparcos stars (HIP 43465, 43491) apparent members of the cluster (Baumgardt et al. 2000). According to this study, the absolute proper motion for M 67 is $\mu_{\alpha} \cos \delta=-6.5$ and $\mu_{\delta}=-6.3$ mas $\mathrm{yr}^{-1}$. Although a few background galaxies are visible in our images, they are not suitable for deriving the correction needed to convert our relative proper motions into the absolute proper motions. 
Table 3. Radial velocity observations.

\begin{tabular}{lcccc}
\hline \hline Set-up & $\Delta \lambda(\AA)$ & $R$ & Plates & OBS-DATE \\
\hline HR02 & $3854-4049$ & 19600 & 2 & $19 / 12 / 2002$ \\
HR14 & $6383-6626$ & 28800 & 5 & $26-28 / 02 / 03$ \\
HR15 & $6607-6965$ & 19300 & 6 & $26-28 / 02 / 03$ \\
HRN15 & $6470-6790$ & 17000 & 3 & $06-11-23 / 02 / 07$ \\
\hline
\end{tabular}

\subsection{Radial velocities: reduction and calibration}

With the aim of obtaining additional membership information for stars in the region of $\mathrm{M}$ 67, we searched the ESO archive (http: //archive.eso.org/) for sets of FLAMES+GIRAFFE@VLT (MEDUSA mode) observations taken simultaneously with a calibration lamp. The MEDUSA mode allows obtaining about 130 spectra at once over a field-of-view of 25 arcmin in diameter. We found observations performed with three different set-ups (HR02, HR14, HR15).

In addition to these data sets, we used the data (HR15N) obtained for a different project in DDT time (278.D-5027). They were collected to determine bona fide possible single solar-type members in M 67 closely resembling the Sun. The aim of this program is to find solar analogues among the M 67 solar type stars. Full analysis of the data is in progress and will be reported elsewhere (Biazzo et al., submitted to A\&A).

The instrumental set-up for each run is given in Table 3, indicating the wavelength range, resolution, the number of plates, and the date of observations.

The spectroscopic data were reduced using the GIRAFFE pipeline GIRBLDRS ${ }^{4}$, in which the spectra were de-biased, flatfield corrected, extracted, and wavelength-calibrated, using both prior and simultaneous calibration-lamp spectra. We also used the gyCrossC.py utility of the GIRAFFE pipeline to measure heliocentric radial velocity $\left(V_{\mathrm{R}}\right)$. For each set-up, we used an appropriate $\mathrm{K} 0$ type template spectra developed by the GIRAFFE pipeline team.

The formal errors reported in the catalog are merely the output of pipeline, which is clearly underestimating the true errors. Nevertheless these estimates retain the information on the goodness of the fit of the cross-correlation function.

There are signs of systematic errors in $V_{\mathrm{R}}$, if repeated measurements of radial velocity from different set-ups are considered. To estimate the amount of such errors, we computed the rms for all determinations of $V_{\mathrm{R}}$ of the same star and averaged that estimate for all multiple measurements of radial velocity. As the result, a single measurement of radial velocity could be affected by systematics reaching up to $\sim 0.6 \mathrm{~km} \mathrm{~s}^{-1}$. Perhaps this is an upper limit to systematic offsets because some stars may have a physical explanation for $V_{\mathrm{R}}$ variations. To estimate the real uncertainty in radial velocities, we suggest summing up this estimate and the formal fitting error in quadrature.

The heliocentric radial velocities and relative errors are shown in Fig. 3 as a function of $V$ magnitude. Several stars have a radial velocity that is very different from the cluster's mean velocity. This can be due to the field stars contaminating the spectroscopic sample or to short-period spectroscopic binaries that are bona fide cluster members. We determined the median $V_{R}$ of our sample of radial velocities and then estimated the dispersion around this median $V_{\mathrm{R}}$, assuming a Gaussian distribution and considering the 68 th percentile from the median. Then we

\footnotetext{
${ }^{4}$ Blecha et al. (2000). See http://discretionary-girbldrs. sourceforge.net/, for GIRAFFE pipeline, software and documentation.
}

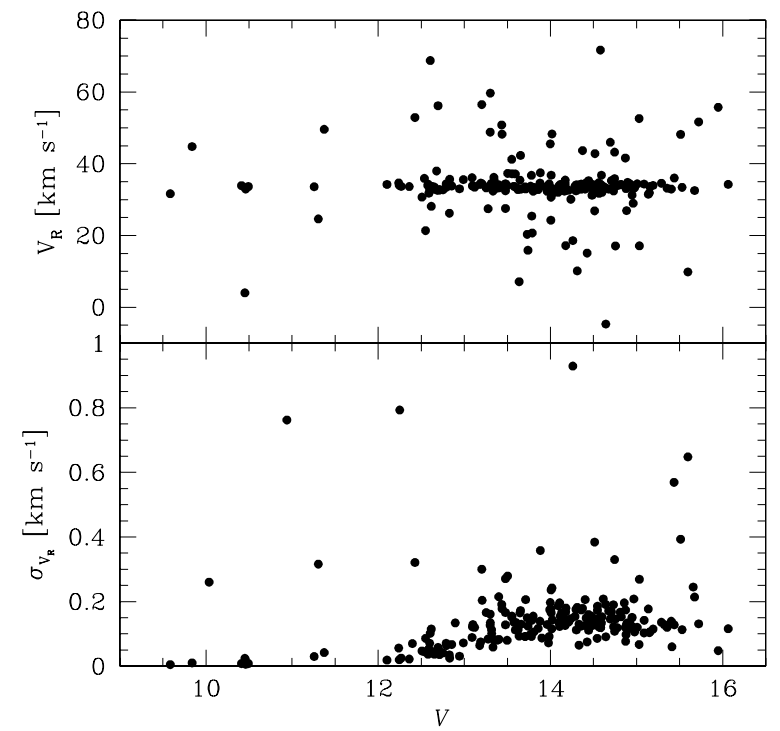

Fig. 3. Heliocentric radial velocities (top panel) and their pipelineerrors (bottom panel) of stars from the spectroscopic sample, plotted as a function of $V$ magnitude.

Table 4. Mean weighted radial velocities for M 67 derived from the data in WEBDA.

\begin{tabular}{ccl}
\hline \hline$\overline{V_{\mathrm{R}}}\left[\mathrm{km} \mathrm{s}^{-1}\right]$ & $N_{s}$ & Reference \\
\hline $34.07 \pm 0.10$ & 144 & Mathieu et al. (1986) \\
$34.77 \pm 0.21$ & 50 & Glushkova et al. (1990) \\
$33.54 \pm 0.13$ & 27 & Glushkova, unpublished \\
$32.89 \pm 0.18$ & 10 & Shetrone et al. (2000) \\
$33.85 \pm 0.16$ & 26 & Melo et al. (2001) \\
$33.67 \pm 0.09$ & 141 & this work \\
\hline
\end{tabular}

iteratively used a 3.5- $\sigma$ clipping to achieve a convergence. The final mean heliocentric radial velocity for M 67 estimated from 141 cluster members is $\overline{V_{R}}=33.67 \pm 0.09 \mathrm{~km} \mathrm{~s}^{-1}$. As indicated by Table 4 , our value of $\overline{V_{\mathrm{R}}}$ is consistent with those derived by the other authors.

\section{Cluster CMD decontamination}

In this section we probe the effectiveness of our proper motions in separating clusters stars from the field stars using a vectorpoint diagram (VPD) in combination with a color-magnitude diagram (CMD).

For stars with $B V$ photometry, in Fig. 4 we show the vectorpoint diagram (top panels) and the CMDs in the $(B-V), V$ plane (bottom panels). In the left panels, there is the whole sample of stars: the middle panels display what we consider to be probable cluster members, the right ones predominantly show the field stars.

In vector-point diagrams around the cluster centroid, we drew a circle with the radius of 6 mas $^{-1} r^{-1}$. Provisionally, we define all points in VPD within this circle as cluster members. The chosen radius is a compromise between losing cluster members with poor proper motions and including field stars sharing the cluster's mean proper motion. Even this crude division into cluster and field stars demonstrates the power of the proper motions derived in this study. A formal determination of membership probability is given in Sect. 4.

As expected, the fainter stars have less well-measured proper motions because of a rapidly decreasing signal-to-noise ratio. 
Table 5. First lines of the catalog available electronically at the CDS.

\begin{tabular}{|c|c|c|c|c|c|c|c|c|c|c|c|c|c|c|c|c|c|}
\hline $\begin{array}{l}\text { ID } \\
\text { (1) }\end{array}$ & $\begin{array}{c}\alpha \\
(2)\end{array}$ & $\begin{array}{c}\delta \\
(3)\end{array}$ & $\begin{array}{c}x \\
(4)\end{array}$ & $\begin{array}{c}y \\
(5)\end{array}$ & $\begin{array}{c}B \\
(6)\end{array}$ & $\begin{array}{l}\sigma_{B} \\
(7)\end{array}$ & $\begin{array}{c}V \\
(8)\end{array}$ & $\begin{array}{l}\sigma_{V} \\
(9)\end{array}$ & $\begin{array}{c}I \\
(10)\end{array}$ & $\begin{array}{c}\sigma_{I} \\
(11)\end{array}$ & $\begin{array}{c}\mu_{\alpha} \cos \delta \\
\text { (12) }\end{array}$ & $\begin{array}{c}\sigma_{\mu_{\alpha} \cos \delta} \\
(13)\end{array}$ & $\begin{array}{c}\mu_{\delta} \\
(14)\end{array}$ & $\begin{array}{l}\sigma_{\mu_{\delta}} \\
(15)\end{array}$ & $\begin{array}{c}P_{\mu} \\
(16)\end{array}$ & $\begin{array}{c}V_{\mathrm{R}} \\
(17)\end{array}$ & $\begin{array}{l}\sigma_{V_{R}} \\
(18)\end{array}$ \\
\hline [\#] & {$\left[{ }^{\circ}\right]$} & {$\left[{ }^{\circ}\right]$} & [pixel] & [pixel] & {$[\mathrm{mag}]$} & [mag] & {$[\mathrm{mag}]$} & [mag] & [mag] & [mag] & [mas/yr] & [mas/yr] & [mas/yr] & [mas/yr] & {$[\%]$} & {$\left[\mathrm{km} \mathrm{s}^{-1}\right]$} & {$\left[\mathrm{km} \mathrm{s}^{-1}\right]$} \\
\hline 1 & 132.897815 & 11.575597 & 3524.156 & -246.966 & 99.999 & 0.999 & 16.064 & 0.009 & 99.999 & 0.999 & 1.73 & 3.51 & 1.73 & 1.25 & 95 & 34.251 & 0.116 \\
\hline 2 & 132.867489 & 11.576494 & 3973.470 & -233.047 & 99.999 & 0.999 & 20.236 & 0.040 & 99.999 & 0.999 & 0.06 & 3.21 & -1.25 & 3.57 & 39 & 999.999 & 999.999 \\
\hline 3 & 133.092502 & 11.576703 & 639.898 & -230.841 & 99.999 & 0.999 & 21.698 & 0.1 & 99.999 & & 13.86 & & & & 29 & 999.999 & \\
\hline 4 & 132.874183 & 11.577474 & 3874.268 & -218.312 & 99.999 & 0.999 & 18.996 & 0.013 & 99.999 & 0.999 & 7.14 & 6.31 & -7.26 & 5.00 & 2 & 999.999 & 999.999 \\
\hline 5 & 133.093782 & 11.577612 & 620.936 & -217.087 & 99.999 & 0.999 & 21.452 & 0.260 & 99.999 & 0.999 & 22.37 & 7.79 & -22.55 & 13.39 & 2 & 999.999 & 999.999 \\
\hline 6 & 132.773239 & 11.578593 & 5369.865 & -199.708 & 99.999 & 0.999 & 17.234 & 0.010 & 99.999 & 0.999 & 25.23 & 6 . & -29.33 & 28.44 & 0 & 999.999 & 999.999 \\
\hline 7 & 132.614021 & 11.578638 & 7728.562 & -194.667 & 99.999 & 0.999 & 18.647 & 0.008 & 99.999 & 0.999 & 5.41 & 0.24 & -2.62 & 2.38 & 28 & 999.999 & 999.999 \\
\hline 8 & 133.035261 & 11.579114 & 1487.807 & -194.539 & 99.999 & 0.999 & 20.867 & 0.046 & 99.999 & 0.999 & 15.05 & 24.16 & 5.71 & 2.32 & 7 & 999.999 & 999.999 \\
\hline 9 & 132.949617 & 11.579316 & 2756.606 & -191.207 & 99.999 & 0.999 & 15.411 & 0.009 & 99.999 & 0.999 & 18.39 & 1.25 & 2.32 & 5.06 & 0 & 90.264 & 0.060 \\
\hline 10 & 132.829375 & 11.579519 & 4538.135 & -186.755 & 99.999 & 0.999 & 19.515 & 0.042 & 99.999 & 0.999 & 6.31 & 6.19 & -13.15 & 10.00 & 0 & 999.999 & 999.999 \\
\hline
\end{tabular}

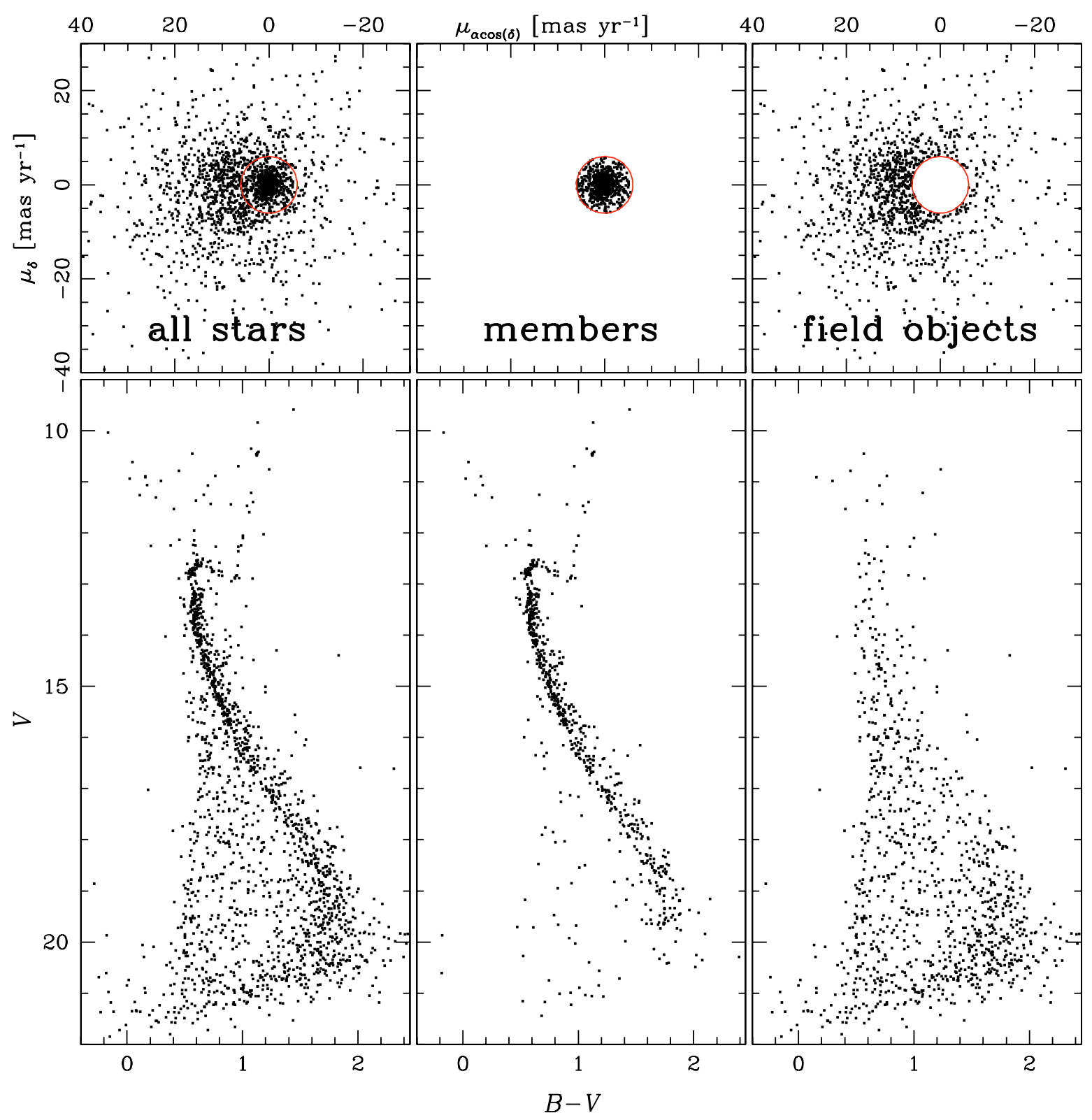

Fig. 4. (Top panels) Proper motion vector-point diagram. Zero point in VPD is the mean motion of cluster stars. (Bottom panels) Calibrated $(B-V)$

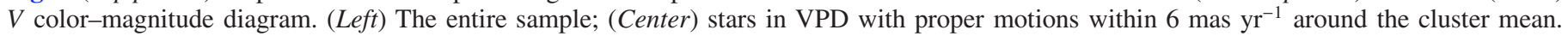
(right) Probable background/foreground field stars in the area of M 67 studied in this paper. All plots show only stars with proper motion $\sigma$ less than $\sim 20$ mas $^{-1} r^{-1}$ in each coordinate. 

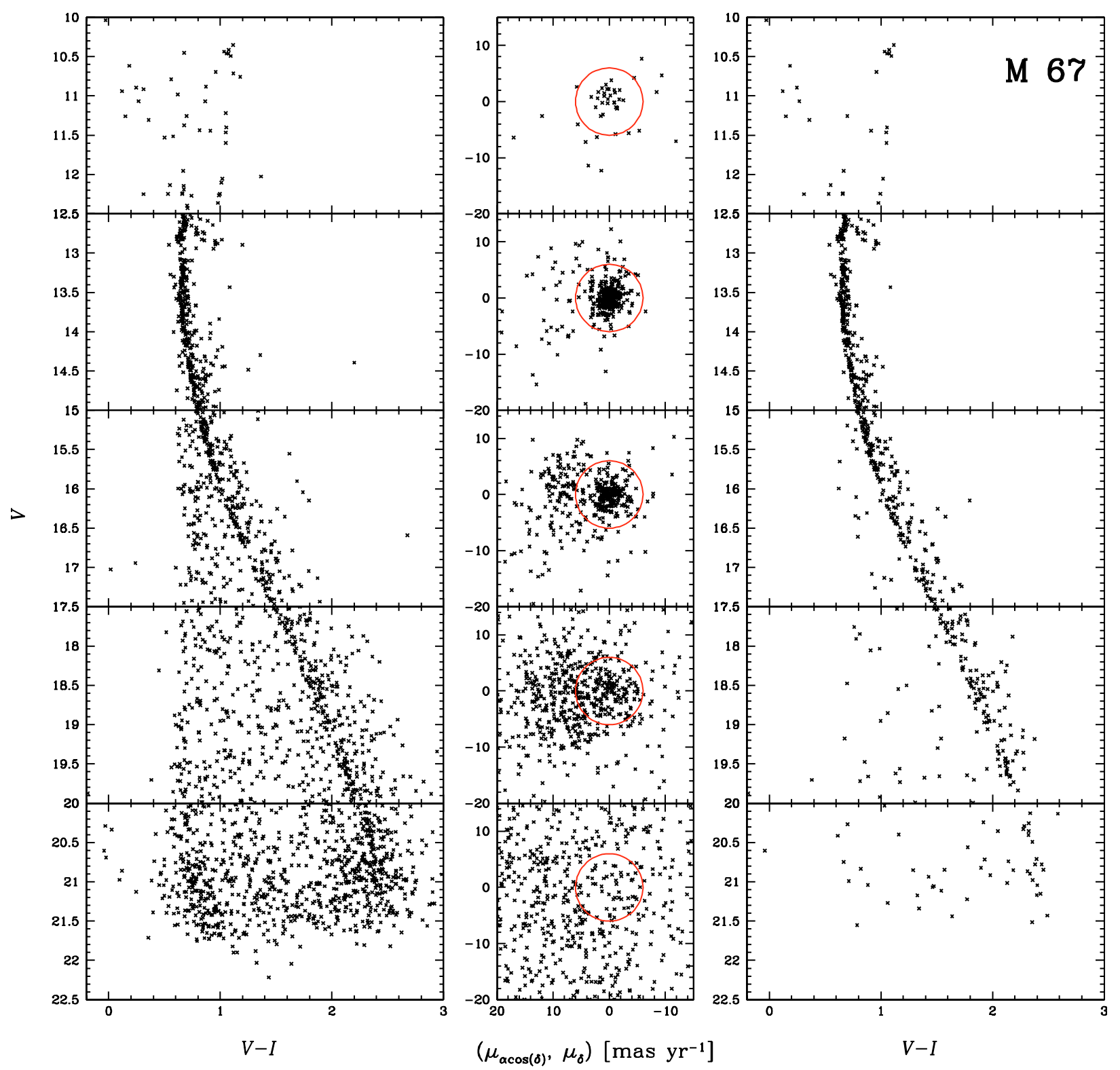

Fig. 5. (Left): color-magnitude diagram for stars with proper motions. (Middle): vector-point diagram for the same stars in corresponding magnitude intervals. A circle in each plot shows the adopted membership criterion at 6 mas $^{-1}{ }^{-1}$. (Right): color-magnitude diagram for stars assumed to be cluster members.

Figure 5 illustrates this fact in the $(V-I), I$ plane binned along the magnitude axis. We note that an acceptable membership is established even for saturated stars at $V \lesssim 12.5$. Note that photometry for bright stars is only derived from short exposures and then adjusted via the zero-point correction to photometry from long exposures.

The separation between the cluster members and field stars is not as obvious as it is for the globular clusters presented in Paper I. In the case of M 67, the distributions of cluster and field proper motions have a substantial overlap, separated only by $\sim 8$ mas $\mathrm{y}^{-1}$. Therefore it is not surprising that at the faint magnitudes where proper motion errors are large, the separation between the cluster and field is poor.

Finally, we note that our field star CMD (right lower panel in Fig. 4) shows no trace of the anomalous clump detected by Balaguer-Núñez et al. (2007) in in the $V, b-y$ and $V, v-y$ color-magnitude diagrams. This is perhaps not surprising because these authors cover a larger area around M 67 than our study does and, according to Balaguer-Núñez et al., the stars in this clump mysteriously avoid the central parts of M 67.

\section{Determination of membership probability}

The vector-point diagram (Fig. 4) of our proper motions clearly shows two populations: a tight clump at $\mu_{\alpha} \cos \delta=\mu_{\delta}=$ 0.0 mas $\mathrm{yr}^{-1}$ mainly representing the cluster stars and an overlapping broad distribution of field stars. Vasilevskis et al. (1958) were the first to formulate the proper motion membership probability as

$P_{\mu}=\frac{\Phi_{\mathrm{c}}}{\Phi_{\mathrm{c}}+\Phi_{\mathrm{f}}}$

where $\Phi_{\mathrm{c}}$ is the distribution of cluster stars and $\Phi_{\mathrm{f}}-$ the distribution of field stars in the vector-point diagram. Usually, these distributions are represented by elliptical normal frequency distributions, i.e., Gaussians, although in some special cases the field 


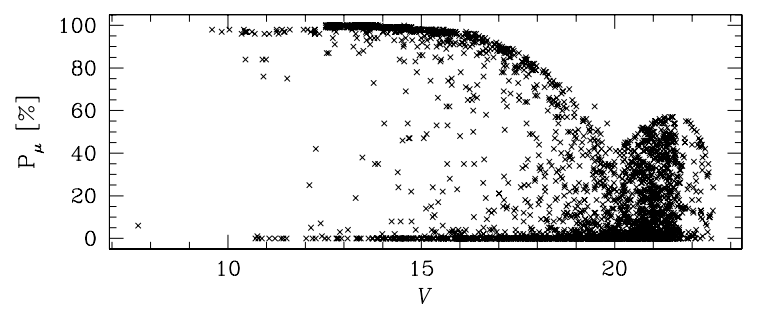

Fig. 6. Membership probabilities as function of the $V$ magnitude, for all the stars in our catalog.

star distribution can locally be adopted as non-Gaussian (Jones 1997; Platais et al. 2001).

There are numerous papers discussing various aspects of calculating the parameters of these distributions and membership probabilities in general; e.g., Jones (1997); Girard et al. 1989; Balaguer-Núñez et al. (2007) and references therein. For membership probability calculations, we chose the local sample method, which is equally reliable in rich open clusters such as NGC 188 (Platais et al. 2003), as well as in sparse clusters like IC 2391 (Platais et al. 2007). In this method, a local sample of stars is selected to represent the properties of a target star as closely as possible. It assures a smooth transition of calculated membership probabilities as a function of magnitude and also accounts for the changes in the magnitude-dependent cluster-tofield star ratio. It has been recognized that a star's brightness and spatial location are the two critical parameters to be considered in the membership probability calculations (Girard et al. 1989; Jones 1997). To counter the effect of a changing clusterto-field star ratio, we chose a sliding 2.5-mag-wide magnitude bin centered on the target. The exception are the extremes of the magnitude range where a magnitude bin is fixed at $2.5 \mathrm{mag}$. The spatial distribution was ignored due to the relatively small size of our field $\left(\sim 30^{\prime} \times 30^{\prime}\right)$ with respect to the radial extent of M 67 out to at least one degree (Sanders 1977). Another component of our approach is to parameterize the magnitude dependence of proper motion dispersions for cluster and field stars. That can be done using photometrically selected samples of cluster and field stars (Fig. 5). Finally, the center of proper motion distribution for cluster stars was adopted at $\mu_{\alpha} \cos \delta=\mu_{\delta}=0.0$ mas $\mathrm{yr}^{-1}$, but for field stars it was estimated from the photometrically selected field stars and also parameterized as a function of magnitude. It should be noted that in the $\mu_{\alpha} \cos \delta$-coordinate, the center of field star distribution in the VPD is at $\sim+6$ mas $\mathrm{yr}^{-1}$, which is significantly larger than reported by Girard et al. (1989). We made no effort to account for the individual proper motion uncertainties other than the parameterizations mentioned above. Poorly measured proper motions, i.e., with uncertainties exceed-

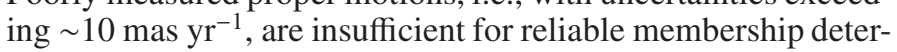
mination in M 67. Nevertheless, we kept these stars to demonstrate the achieved accuracies over the entire magnitude range.

Calculation of the two remaining parameters (heights of Gaussian peaks) needed to fully characterize the cluster and field distributions in the VPD is straightforward (Platais et al. 2003). We note that the distribution of field stars appears to be nearly circular, which further simplifies the calculations. The distribution of membership probabilities (Fig. 6) in effect reflects the degree of reliability of our proper motions. Thus, the separation between the cluster and field is convincing for $V<18$. In the range $18<V<20$ the decreasing maximum $P_{\mu}$ indicates a steadily growing contamination by field stars. The unexpected rise in maximum $P_{\mu}$ at $V>20$ is an artifact caused by a nearly total blending of both cluster and field proper motion distributions.
In other words, in this magnitude range the accuracy of proper motions is inadequate for reliable calculations of membership probability.

\section{Comparison with existing photometric data}

In Fig. 7, we show color-magnitude diagram in the $[(B-V), V]$ and $[(V-I), I]$ planes for those stars of our sample that have a membership probability $P_{\mu}>60 \%$ (see Sect. 4 for details). The saturation level of deep $V$ exposures is indicated by the dotted line. The stars from the secondary standard catalog by Stetson $(2000,2005)$ are over-plotted as red asterisks. The cyan color-coded circles show the location of the Sandquist (2004) single stars. As expected, the location of main sequence from our photometry and that of Sandquist is nearly identical because our photometry was calibrated using Sandquist's data. The upper main sequence and giant branch in $[(B-V), V]$ have some small offsets, if different sources are considered. In part, that can be explained by retaining single stars only in the Sandquist photometry.

In Fig. 8 we show the photometric residuals as a function of magnitude and color for the common stars between Sandquist and our data. Sandquist (2004) presents extensive comparisons with other photometric data from the literature, in particular, with Montgomery et al. (1993), so we refer the reader to this study for detailed comparisons. The referee suggested making a request for the Ben Taylor's new improved VRI photometry and performing a direct comparison with this new catalog. Ben Taylor kindly released his photometry to us and this comparison is shown in Fig. 9. If there are any offsets, then they are certainly less than 0.01 mag in both $V$ magnitude and $V-I$ color.

\section{Comparison with theoretical isochrones}

The CMDs of Galactic open clusters have long been used as tests of stellar models for intermediate-mass stars. The most important issue is related to the long standing problem of the real extension of the convective core during the central H-burning stage. How much larger is the convective core extension than the canonical prediction provided by the classical Schwarzschild criterion; i.e., what is the amount of convective core overshooting (Sandquist 2004; Cassisi 2002, and references therein)?

Open cluster studies have shown that stellar models must allow for the occurrence of convective core overshooting in order to provide a satisfactory match to the observed CMDs (see Kalirai et al. 2001, and references therein). On the other hand, a still unsettled issue is related to how convective core overshooting has to decrease in real stars when the size of the canonical convective core decreases with the decreasing total stellar mass (e.g., Woo \& Demarque 2001). In the majority of Galactic open clusters, this is not a big problem. However, an age difference exceeding \pm 1 Gyr with respect to the age of M 67 is sufficient for stars to enter the evolutionary phase with a very extended convective core, even encroaching the mass range normally having a radiative core. In the case of $\mathrm{M} 67$, the mass of stars at the main sequence turnoff (TO) is $\sim 1.2 M_{\odot}$, which is in the critical mass interval where the amount of overshooting is decreasing with mass. Therefore, M 67 is crucial for exploring the efficiency of convective core overshoot in stars whose mass is high enough to have a convective core but not high enough to allow for a very extended convective core.

For this reason, M 67 has been the subject of many detailed studies such as those recently performed by Sandquist (2004); 
R. K. S. Yadav et al.: Ground-based CCD astrometry with wide-field imagers. II.

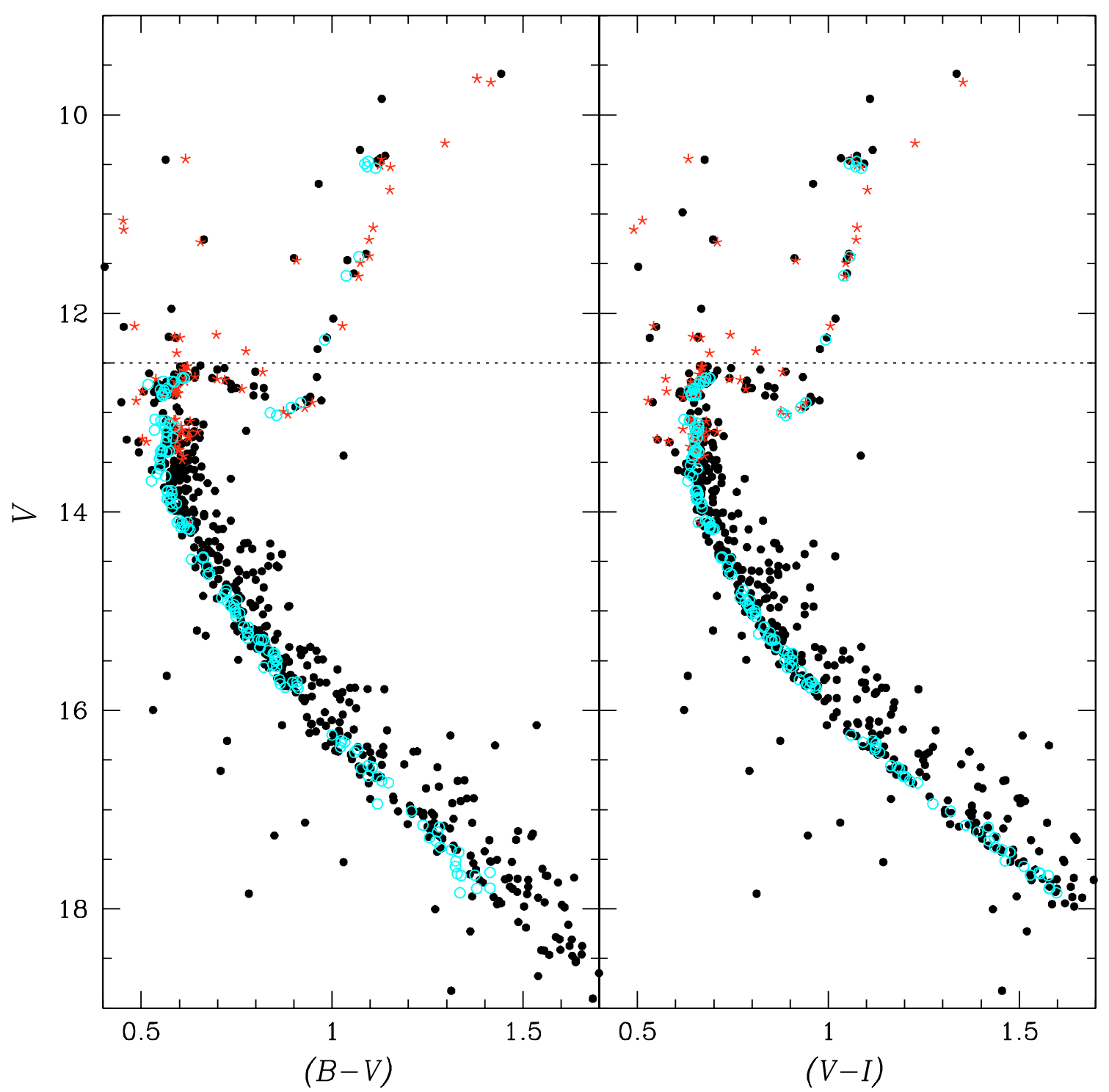

Fig. 7. Comparison of our photometry (filled circles) with Stetson's (2000, 2005; marked by asterisks) and from Sandquist (2004; marked by the open circles).

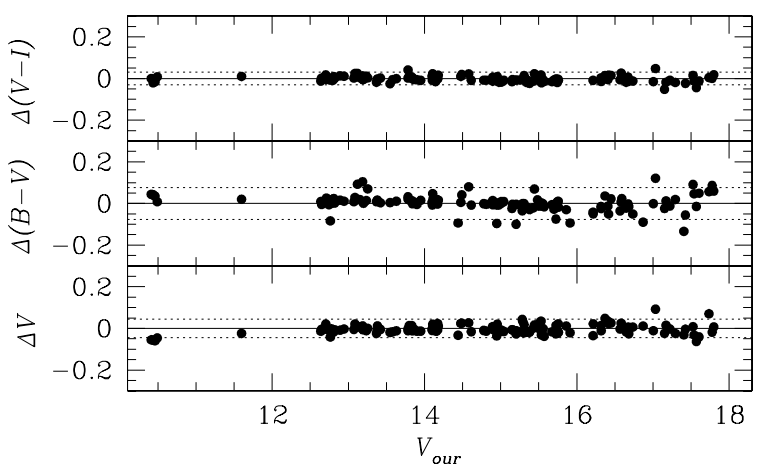

Fig. 8. Star-by-star comparison of our photometry with Sandquist (2004).

VandenBerg \& Stetson (2004) and VandenBerg et al. (2007). The work of VandenBerg \& Stetson (2004) was specifically devoted to constraining the efficiency of convective core overshooting in stars whose mass is similar to that of the stars currently at the TO of M 67. Their main conclusion - also supported by the

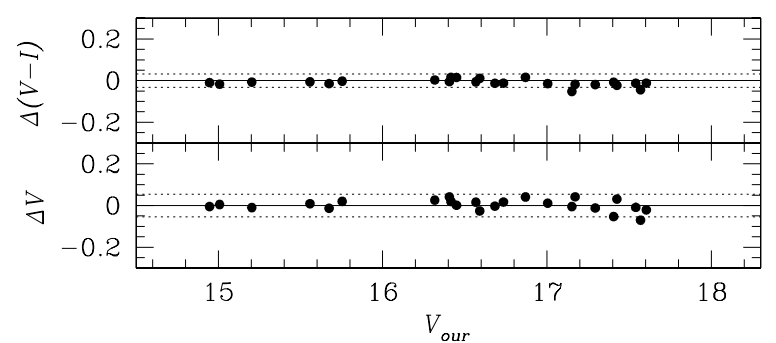

Fig. 9. Comparison of our photometry with the one by Taylor (2008).

results shown by Pietrinferni et al. (2004) - is that the extension of overshooting region has to be very small but not zero for masses of about $1.2 M_{\odot}$, at least for solar metallicity.

A detailed discussion of this issue is clearly beyond the scope of this paper. Nonetheless, a comparison of our observational data for M 67 and the theoretical isochrones available in various independent libraries of stellar models should provide further insight into the capability of commonly-used sets of stellar models in reproducing empirical constraints by CMD of 


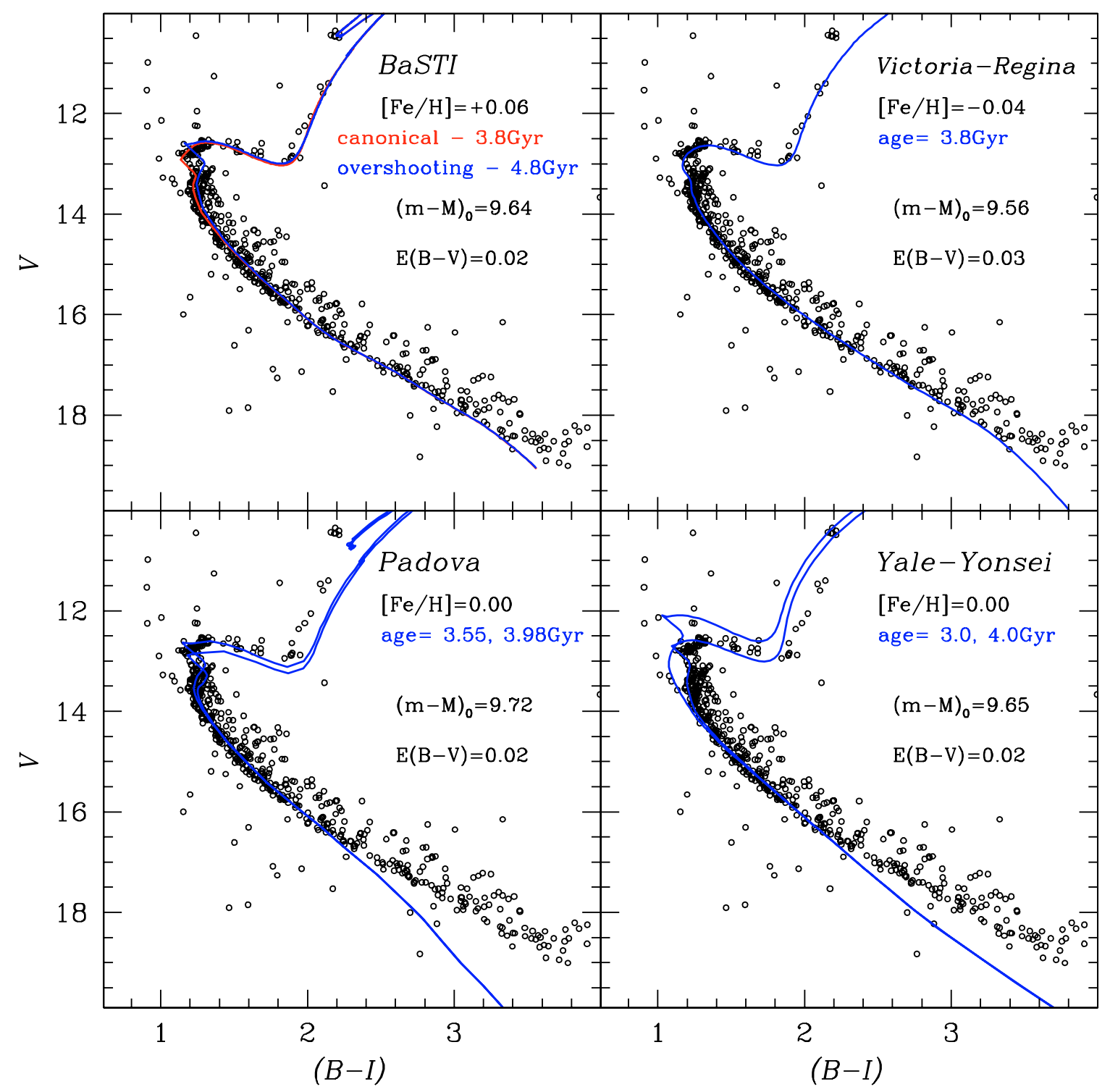

Fig. 10. Comparison of our empirical data with theoretical isochrones from independent libraries of stellar models (see the text for more details). The fit between theory and observations was performed by adopting the labeled values for the true distance modulus and reddening.

the old Galactic open clusters. For this purpose, we adopted the following sets of theoretical tracks: the $\mathrm{BaSTI}^{5}$ models provided by Pietrinferni et al. (2004; see also Cordier et al. 2007), the Padova models published by Girardi et al. (2000), the YonseiYale isochrones provided by Yi et al. (2001) in their second release, and the Victoria-Regina models published by VandenBerg et al. (2006). In the case of the Yonsei-Yale isochrones, we applied their set of isochrones transformed into the observational plane using the $T_{\text {eff-to-color transformation tables provided by }}$ Green et al. (1987).

The comparison among various fits of theoretical isochrones to observational CMDs is shown in Fig. 10. Before discussing this figure, it is necessary to briefly review the best estimates of metallicity, reddening, and distance for M 67. The metallicity of M 67 is essentially solar or maybe slightly lower

\footnotetext{
5 Note added in proof.- a new release of the BaSTI model library is available since March 18th 2008. For more details, we refer the interested reader to the BaSTI archive URL: http://www . oa-teramo. inaf.it/BASTI/
}

(Taylor 2007). This result is well-established on the basis of both high- (Tautvaišiene et al. 2000) and low-resolution spectroscopy (Friel \& Janes 1993). The existing estimates of interstellar extinction in the direction of M 67 have been recently re-analyzed by Taylor (2007). The most likely mean value of $E(B-V)=0.041 \pm 0.004$ derived by Taylor is slightly higher than the value suggested by Schlegel et al. (1998) and than those provided by the fits between theoretical isochrones and empirical data (e.g., VandenBerg \& Clem 2003). Given the intrinsic uncertainties in calibration of $E(B-V)$, the true reddening of M 67 could be anywhere from 0.02 to $0.04 \mathrm{mag}$.

As for the distance to M 67, Percival \& Salaris (2003) measured the distance modulus at $(m-M)_{\circ}=9.60 \pm 0.09$, adopting $E(B-V)=0.04 \pm 0.02$ and $[\mathrm{Fe} / \mathrm{H}]=0.02 \pm 0.06$. Their measurement was obtained by applying the MS-fitting technique to the CMD from Montgomery et al. (1993) data by using a sample of local $G$ and $K$ dwarfs with accurate Hipparcos parallaxes and a near-solar metallicity. The Percival \& Salaris (2003) estimate was subsequently confirmed by Sandquist (2004), who obtained the same distance modulus using new photometric data. 

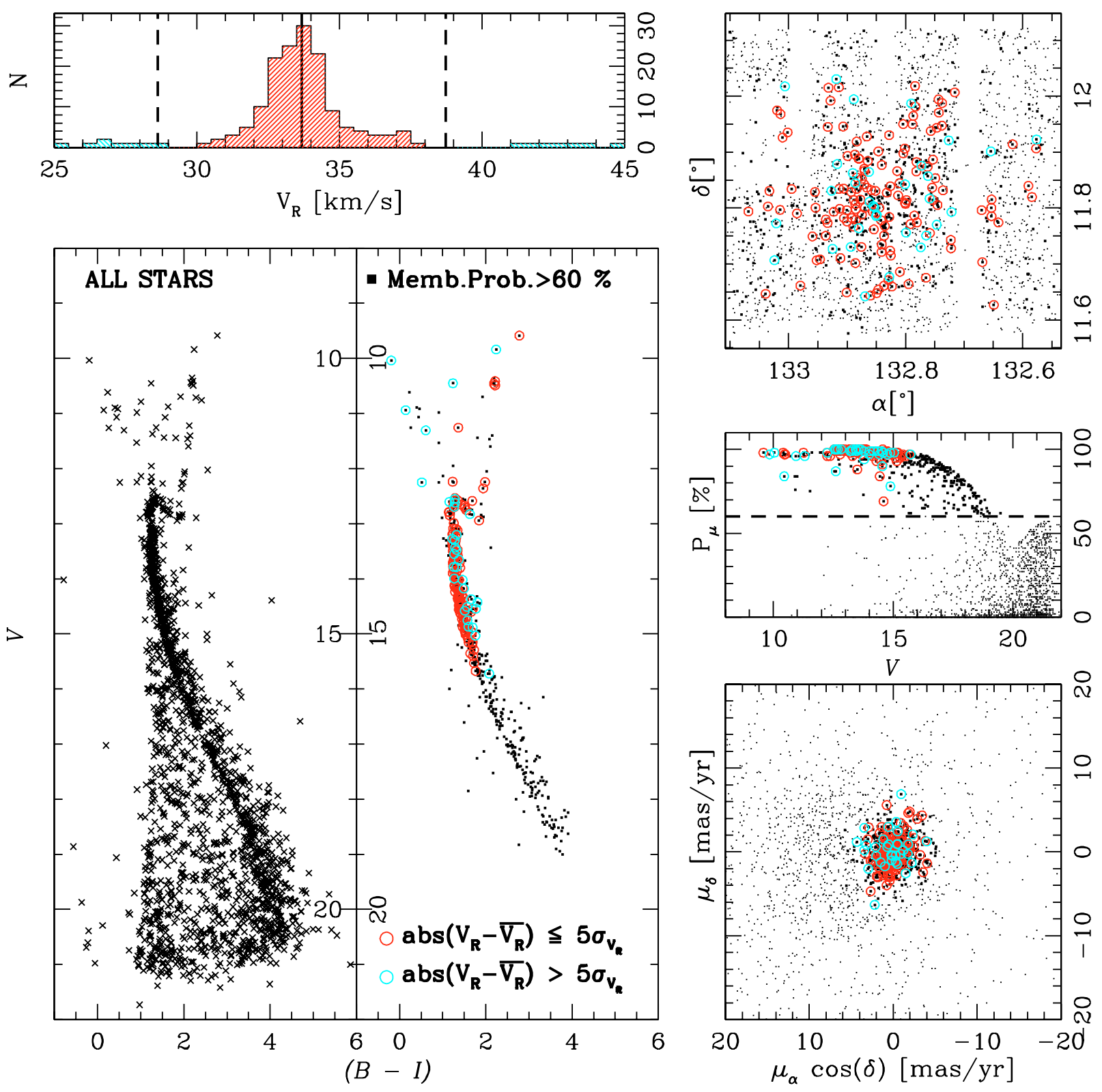

Fig. 11. This plot is a summary of the quantities listed in the catalog. Positions $(\alpha, \delta)$, photometry (in $B, V$, and $I$ ), radial velocities $\left(V_{\mathrm{R}}\right.$ ), proper motions $\left(\mu_{\alpha} \cos \delta, \mu_{\delta}\right)$, and membership probabilities $\left(P_{\mu}\right)$. Thin dots are stars with a $P_{\mu} \leq 60 \%$, for thick dots $\mathrm{P}_{\mu}>60 \%$. Red circles are stars for which $\left\|V_{\mathrm{R}}-\overline{V_{\mathrm{R}}}\right\|<5 \sigma_{V_{\mathrm{R}}}$, if not they are marked in cyan. Note that many of those are potentially short period binaries.

The comparison shown in Fig. 10 is quite informative. Within the errors the BaSTI (Pietrinferni et al. 2004) isochrones with $[\mathrm{Fe} / \mathrm{H}]=0.06$ reproduce the $\mathrm{CMD}$ with $E(B-V)=0.02$ and $(m-M)_{\circ}=9.64$ nicely, in agreement with previous determinations. Both isochrones are shown with and without convective core overshoot. The adopted metallicity appears to be the upper limit of any existing estimate of $[\mathrm{Fe} / \mathrm{H}]$ in $\mathrm{M} 67$. The MS and the RGB appear to match both a canonical and the isochrone with overshoot. We note that the brightness and color of He-burning clump stars are reproduced very well by the BaSTI isochrones for the adopted reddening and distance modulus.

The best fit of main sequence using the Victoria-Regina isochrones (VandenBerg et al. 2006) can be achieved with a distance modulus equal to $9.56 \mathrm{mag}$ and a reddening of 0.03 . The Victoria-Regina isochrones do not follow through the phase of core He burning, hence we cannot test a match to the red clump stars.
Sandquist (2004) has shown that a number of isochrones from the literature are unable to fit the location of MS at $V>$ 15.5. This result is confirmed by a fit of Padova (Girardi et al. 2000) and Yonsei-Yale isochrones (Yi et al. 2001) to our data shown in Fig. 10. As suggested by Sandquist (2004) the available $T_{\text {eff-color transformations are poor for main sequence stars }}$ at $T_{\text {eff }}<5000 \mathrm{~K}$. This deficiency is corrected for VictoriaRegina isochrones by applying semi-empirical color transformations (VandenBerg \& Clem 2003). We note that, in the same $T_{\text {eff }}$ range, the theoretical isochrones do not closely follow the MS stars in metal-poor globular clusters either (Bedin et al. 2001). This can be attributed largely to the same problem in the adopted $T_{\text {eff-color relations. }}$

The main result of our isochrone fit to the new colormagnitude diagram for M 67 is the confirmation of a small convective core overshoot which is required to achieve a close match to the features near the main sequence turnoff region. This result 
is in very good agreement with the earlier results by Sandquist (2004) and VandenBerg \& Stetson (2004). We would like to point out that in this study we have performed comparisons between the theory and observations by employing four independent and updated libraries of stellar models.

\section{The catalog}

The catalog is available electronically at Astronomy \& Astrophysics (or also under request to the authors). In the catalog, Col. (1) contains the running number; Cols. (2) and (3) give the J2000 equatorial coordinates in decimal degrees for the epoch J2000.13, while Cols. (4) and (5) provide the pixel coordinates $x$ and $y$ in a distortion-corrected reference frame. Colums (6) to (11) give photometric data, i.e., $B, V, I$ magnitudes and their errors. If photometry in a specific band is not available, a flag equal to 99.999 is set for the magnitude and 0.999 - for the error. The next four Cols. (12)-(15) give relative proper motions and their standard errors. Column (16) gives the proper motion membership probability $P_{\mu}$. The last two Cols. (17) and (18) give radial velocity $\left(V_{\mathrm{R}}\right)$ and its error, if available; otherwise, the table reports 999.999.

Figure 11 shows a summary of the basic properties of stars in the catalog. Here we have plotted a histogram of radial velocities, the spatial distribution of all stars, color-magnitude diagrams, the distribution of membership probabilities, and a vector-point diagram. They provide additional information on similar plots in the previous figures.

\section{Conclusions}

We have applied the techniques developed by Anderson et al. (2006, Paper I) to a cornerstone open cluster, M 67. We demonstrate that the CCD observations taken just four years apart can provide accurate proper motions, aiding in decontaminating the cluster CMD down to $V \sim 20$. An open issue with these open clusters is the intrinsically low spatial density of cluster members, especially in the outer parts of the clusters, which makes it difficult to apply the local-transformation approach rigorously. We believed that, with a geometrically stable CCD mosaic (the WFI@2.2 $\mathrm{m}$ is apparently not so), it would be possible to use the global plate solutions for astrometry and avoid the problem of an inadequate local reference frame.

The high precision of our astrometry and $B, V, I$ photometry once again underscores the importance of accurately representing PSF across the entire field-of-view, exemplified by the concept of empirical PSF (Paper I). Our new photometry confirms that the convective core overshoot in M 67 should be small, as already pointed out by Sandquist (2004) and VandenBerg \& Stetson (2004).

A substantial addition to the existing data sets are our proper motions at $V>16$, up to 5 mag deeper than the published proper motion catalogs. High-precision radial velocities for 211 stars down to $V=16$ are also helping to establish a list of bona fide cluster members in M 67. One of the goals of this paper is to share the new data with the astronomical community prior to comprehensively analyzing and finalizing of cluster membership at faint magnitudes.

Acknowledgements. R. K. S. Yadav is grateful to the University of Padova for the grant that made this Indo-Italian collaboration possible. I. Platais gratefully acknowledges support from the National Science Foundation through grant
AST 04-06689 to Johns Hopkins University. We thank the anonymous referee for careful reading and useful suggestions. We also thank Ben Taylor for providing us with their new unpublished catalog in electronic format.

\section{References}

Anderson, J., Bedin, L. R., Piotto, G., Yadav, R. K. S., \& Bellini, A. 2006, A\&A, 454, 1029 (Paper I)

Balaguer-Núñez, L., Galadí-Enríquez, D., \& Jordi, C. 2007, A\&A, 470, 585

Baumgart, H., Dettbarn, C., \& Wielen, R. 2000, A\&AS, 146, 251

Bedin, L. R., Anderson, J., King, I. R., \& Piotto, G. 2001, ApJ, 560, L75

Biazzo, K., Pasquini, L., Girardi, L., et al. 2007, A\&A, 475, 981

Blecha, A., Cayatte, V., North, P., Royer, F., \& Simond, G. 2000, Proc. SPIE, 4008, 467

Cassisi, S., Salaris, M., \& Bono, G. 2002, ApJ, 565, 1231

Cordier, D., Pietrinferni, A., Cassisi, S., \& Salaris, M. 2007, AJ, 133, 468

Dupree, A. K., Whitney, B. A., \& Pasquini, L. 1999, ApJ, 520, 751

Friel, E. D., \& Janes, K. A. 1993, A\&A, 267, 75

Gilliland, R. L., Brown, T. M., Duncan, D. K., et al. 1991, AJ, 101, 541

Girard, T. M., Grundy, W. M., López, C. E., \& van Altena, W. F. 1989, AJ, 98, 227

Girardi, L., Bressan, A., Bertelli, G., \& Chiosi, C. 2000, A\&AS, 141, 371

Glushkova, E. V., \& Rastorguev, A. S. 1990, AZh, 34, 674

Green, E. M., Demarque, P., \& King, C. R. 1987, The Revised Yale Isochrones and Luminosity Functions (New Haven: Yale Univ. Obs.)

Jones, B. F. 1997, Mem. SAI, 68, 833

Jones, B. F., Fischer, D., \& Soderblom, D. R. 1999, AJ, 117, 330

Kalirai, J. S., Richer, H. B., Fahlman, G. G., et al. 2001, AJ, 122, 266

Landolt, A. U. 1992, AJ, 104, 340

Lattanzi, M. G. 1993, in Workshop on Databases for Galactic Structure, ed. A. G. Davis Philip, B. Hauck, \& A. R. Upgren, 173

Mathieu, R. D., Latham, D. W., Griffin, R. F., \& Gunn, J. E. 1986, AJ, 92, 1100 Mathieu, R. D., Latham, D. W., \& Milone, A. E. 1997, in The Third Pacific Rim Conference on Recent Development of Binary Star Research, ed. K.-C. Leung, ASP Conf. Ser. 130, 113

Mathieu, R. D., van den Berg, M., Torres, G., et al. 2003, AJ, 125, 246

Melo, C. H. F., Pasquini, L., \& De Medeiros, J. R. 2001, A\&A, 375, 851

Momany, Y., Vandame, B., Zaggia, S., et al. 2001, A\&A, 379, 436

Montgomery, K. A., Marschall, L. A., \& Janes, K. A. 1993, AJ, 106, 181

Pace, G., \& Pasquini, L. 2004, A\&A, 426, 1021

Pasquini, L., \& Belloni, T. 1998, A\&A, 336, 902

Pasquini, L., Belloni, T., \& Abbott, T. M. C. 1994, A\&A, 290, L17

Pasquini, L., Randich, S., \& Pallavicini, R. 1997, A\&A, 325, 535

Percival, S. M., \& Salaris, M. 2003, MNRAS, 343539

Pietrinferni, A., Cassisi, S., Salaris, M., \& Castelli, F. 2004, ApJ, 612, 168

Platais, I., Kozhurina-Platais, V., Barnes, S., et al. 2001, AJ, 122, 1486

Platais, I., Kozhurina-Platais, V., Mathieu, R. D., et al. 2003, AJ, 126, 2922

Platais, I., Melo, C., Mermilliod, J.-C., et al. 2007, A\&A, 461, 509

Randich, S., Sestito, P., Primas, F., Pallavicini, R., \& Pasquini, L. 2006, A\&A, 450,557

Randich, S., Primas, F., Pasquini, L., et al. 2007, A\&A, 469, 163

Richer, H. B., Fahlman, G. G., Rosvick, J., \& Ibata, R. 1998, 504, L91

Sanders, W. L. 1977, A\&AS, 27, 89

Sandquist, E. L. 2004, MNRAS, 347, 101

Schlegel, D. J., Finkbeiner, D. P., \& Davis, M. 1998, 500, 525

Shetrone, M. D., \& Sandquist, E. L. 2000, AJ, 120, 1913

Stello, D., Bruntt, H., Kjeldsen, H., et al. 2007, MNRAS, 377, 584

Stetson, P. B. 2000, PASP 112, 925

Stetson, P. B. 2005, PASP 117, 563

Tautvaišiene, G., Edvardsson, B., Tuominen, I., \& Ilyin, I. 2000, A\&A, 360, 499

Taylor, B. J. 2007, AJ, 133, 370

Taylor, B. J., Joner, M. D., \& Jeffery, E. J. 2008, ApJS, 176, in press

Vasilevskis, S., Klemola, A., \& Preston, G. 1958, AJ, 63, 387

VandenBerg, D. A., \& Clem, J. L. 2003, AJ, 126, 778

VandenBerg, D. A., \& Stetson, P. B. 2004, PASP, 116, 997

VandenBerg, D. A., Bergbusch, P. A., \& Dowler, P. D. 2006, ApJS, 162, 375

VandenBerg, D. A., Gustafsson, B., Edvardsson, B., et al. 2007, ApJ, 666, L105

van den Berg, M., Verbunt, F., \& Mathieu, R. D. 1999, A\&A, 347, 866

Woo, J.-H., \& Demarque, P. 2001, AJ, 122, 1602

Yi, S., Demarque, P., Kim, Y.-C., et al. 2001, ApJS, 136, 417

Zacharias, N., Urban, S. E., Zacharias, M. I., et al. 2004, AJ, 127, 3043

Zhao, J. L., Tian, K. P., Pan, R. S., He, Y. P., \& Shi, H. M. 1993, A\&AS, 100, 243 